\title{
Gastric adenosquamous carcinoma producing granulocyte-colony stimulating factor
}

\author{
Kazuya Endo ${ }^{1}$, Shunji Kohnoe ${ }^{1}$, Takeshi Okamura ${ }^{2}$, Masaru Haraguchi ${ }^{2}$, Eisuke Adachi ${ }^{2}$, Yasushi Toh ${ }^{2}$, \\ Hideo Baba ${ }^{3}$, and Yoshihiko Maehara ${ }^{3}$ \\ ${ }^{1}$ Department of General Surgery, Fukuoka Dental College Hospital, 2-15-1 Tamura, Sawara-ku, Fukuoka 814-0193, Japan \\ ${ }^{2}$ Department of Gastroenterologic Surgery, National Kyushu Cancer Center, Fukuoka, Japan \\ ${ }^{3}$ Department of General Surgery, Graduate School of Medical Science, Kyushu University, Fukuoka, Japan
}

\begin{abstract}
We report a case of adenosquamous carcinoma of the stomach that produced granulocyte-colony stimulating factor (G-CSF). The patient, who had an admission diagnosis of advanced gastric cancer, had marked leukocytosis without evidence of infection. After leukemia and metastatic leukemoid reaction were excluded by bone marrow examination, a G-CSFproducing cancer was suspected as the cause of the abnormally elevated serum G-CSF level. The resected stomach tumor was histologically diagnosed as adenosquamous carcinoma; positive expression of G-CSF by tumor cells was shown with immunohistochemical detection, which confirmed the preoperative diagnosis. Recurrent disease in the liver and lymph nodes, accompanied by leukocytosis and re-elevation of serum G-CSF, developed just 3 months after the curative gastrectomy and adjuvant chemotherapy. All of the recurrent disease was resected, restoring normal levels of serum G-CSF. The patient survived for almost 2 years after the initial surgery with extensive chemotherapy, including weekly treatment with paclitaxel, before finally succumbing to liver failure secondary to extensive liver metastasis.
\end{abstract}

Key words Granulocyte-colony stimulating factor · Gastric cancer $\cdot$ Chemotherapy $\cdot$ Adenosquamous carcinoma

\section{Introduction}

A striking leukocytosis without evidence of infection is known to occur occasionally in patients with nonhematological neoplasms. The production of granulocyte colony-stimulating factor (G-CSF) has been reported in patients with lung cancer [1], as well as in those with carcinoma of the gallbladder [2], liver [3], esophagus [4], stomach [5], pancreas [6], and thyroid [7]. G-CSF expression has been implicated in the progression of malignant behavior in nonhematopoietic cancers

Offprint requests to: $\mathrm{K}$. Endo

Received: December 27, 2004 / Accepted: March 16, 2005
[4]. Although the frequency of G-CSF-producing tumors is low, adenosquamous carcinoma of the stomach itself is comparatively rare; the incidence of gastric adenosquamous carcinoma has been estimated to range around $0.5 \%$, and the tumor has a poorer prognosis than that of adenocarcinomas originating in other sites.

This report describes a patient with two rare coexisting malignant factors - adenosquamous carcinoma of the stomach that produced G-CSF - who underwent curative gastrectomy but developed early recurrence in the liver, accompanied by pronounced leukocytosis. We improved the prognosis of the patient, while maintaining quality of life, with the combination of an adequate surgical approach and extensive chemotherapy for repeated recurrences in liver and lymph nodes.

\section{Case report}

A 55-year-old Japanese man was admitted to the hospital in August 2001 with the main complaint of general fatigue and anemia. Laboratory testing showed pronounced leukocytosis (WBC, 35000/ $\mu \mathrm{l}$, with $55 \%$ band forms, $42 \%$ segmented forms, and 3\% lymphocytes). Serum concentrations of carbohydrate antigen 19-9, squamous cell carcinoma antigen, and carcinoembryonic antigen were normal. Gastrointestinal endoscopy showed a bulky type- 1 tumor and a continuous type-2 tumor in the gastric antrum (Fig. 1A,B); these were not independent of each other. Biopsy specimens taken during endoscopy were histologically proven to be adenosquamous carcinoma. Computed tomography and echography showed no evidence of metastatic lesions, but did indicate marked perigastric lymphadenopathy (Fig. 1C).

No evidence of dissemination into the peritoneal cavity was seen on barium enema examination. Bone marrow aspirate was hypercellular, with almost normal 

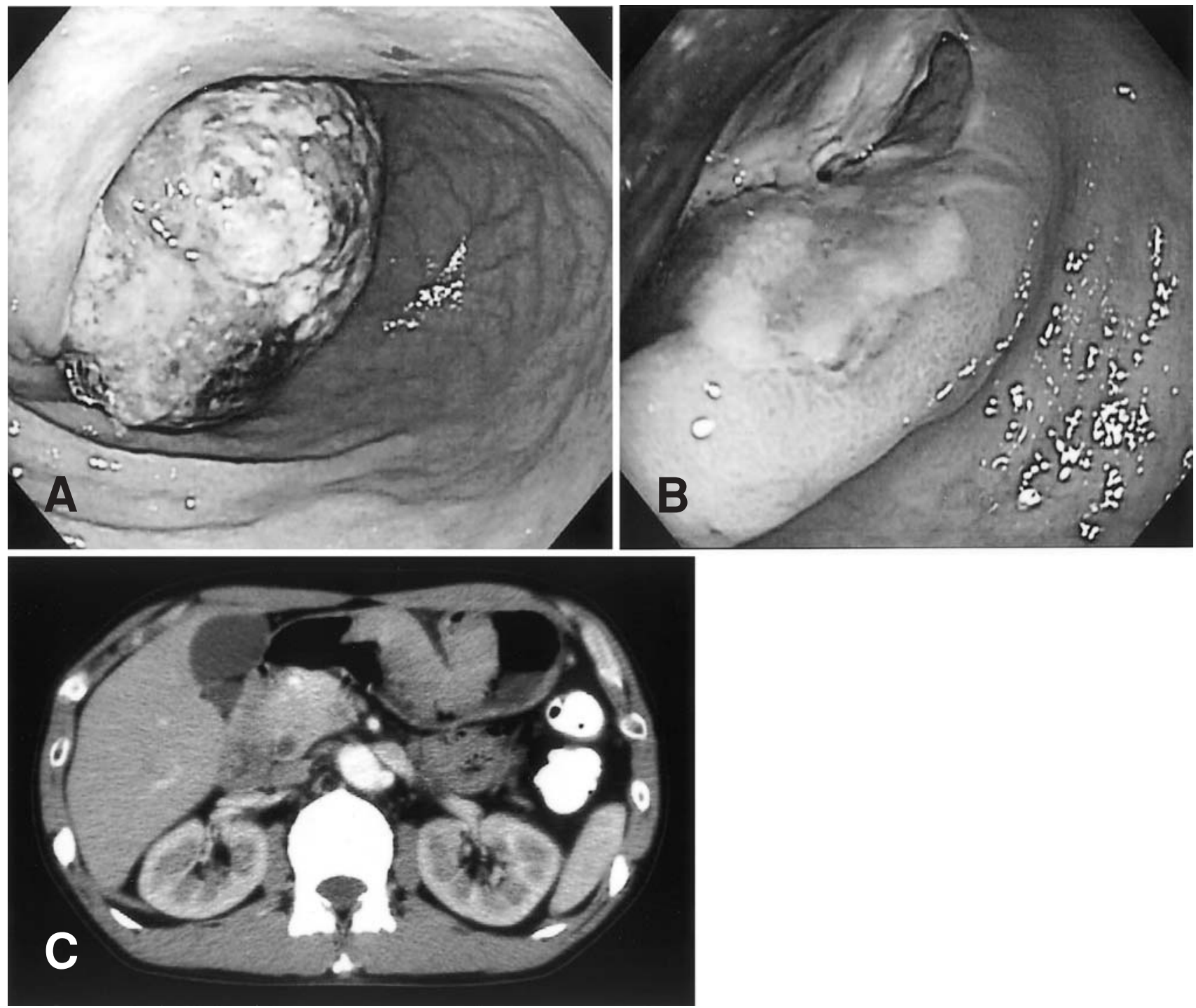

Fig. 1A,B. Initial appearance of tumor on gastrointestinal endoscopy. A bulky type-1 gastric cancer and $\mathbf{B}$ a continuous type-2 lesion in the antrum of the stomach. $\mathbf{C}$ Computed tomography indicated gastric cancer in the antrum, and marked perigastric lymphadenopathy

percentages of various cell types. Cytogenetic examination did not detect a Ph1 chromosome. Serum Creactive protein (CRP) concentration was elevated to $3.5 \mathrm{ng} / \mathrm{ml}$, not a terribly high level, and there were no strong signs of inflammation or infection, except for the marked leukocytosis. Notably, the serum G-CSF concentration was elevated to $105 \mathrm{pg} / \mathrm{ml}$ (normal, below $10 \mathrm{pg} / \mathrm{ml})$ (Table 1).

A partial gastrectomy with extended lymphadenectomy (D3) was performed [8]. It was judged to be a curative procedure because there was no evidence of hepatic metastasis or seeding of the peritoneum. The resected specimen was a large type- 1 tumor, measuring $7.0 \times 8.0 \mathrm{~cm}$ in size, with a type 2 tumor, $4.0 \times 3.5 \mathrm{~cm}$ in size, beside the main lesion (Fig. 2). Histopathological
Table 1. Pre- and postoperative concentrations of serum granulocyte-colony stimulating factor (G-CSF)

\begin{tabular}{lcc}
\hline & \multicolumn{2}{c}{ G-CSF concentration $(\mathrm{pg} / \mathrm{ml})$} \\
\cline { 2 - 3 } & First operation & Second operation \\
\hline Preoperative & 105 & 57 \\
Postoperative & 11 & 10 \\
\hline
\end{tabular}

Normal range, below $10 \mathrm{pg} / \mathrm{ml}$

investigation revealed adenosquamous carcinoma involving the subserosa, with invasion of lymphatic vessels, but without evidence of venous invasion. Although no distant metastasis was recognized, pathologic metastasis was found in dissected lymph nodes. Of 42 dis- 
sected lymph nodes, metastases were recognized in No.5 (1 of 3), No.6 (2 of 5), and No.14v (3 of 6) [8]. According to the TMN classification, the stage of the cancer was T2N2M0 (stage IIIa). After the operation, all symptoms, including fever, general fatigue, and the abnormal leukocytosis improved, and the serum G-CSF concentration decreased to the normal level (Table 1). We investigated G-CSF expression in the resected stomach, using immunohistochemical staining with a monoclonal antibody against G-CSF. Cells of the gastric cancer showed positive staining, in the cytoplasm, for G-CSF (Fig. 3). Although adjuvant chemotherapy was performed with oral S-1 (TS-1), liver recurrence developed just 3 months after the gastrectomy (Fig. 4) along

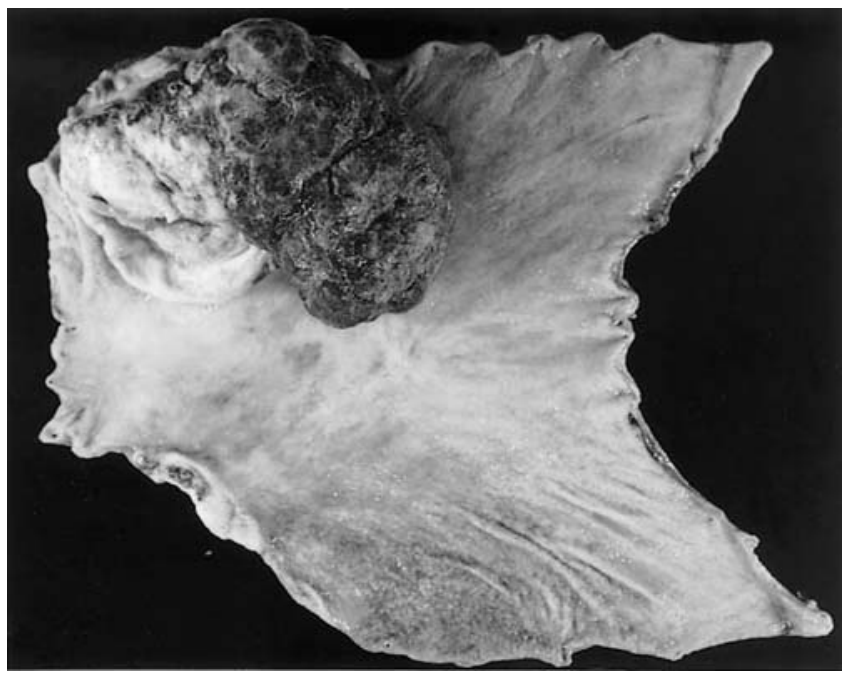

Fig. 2. Macroscopic appearance of resected stomach confirms the preoperative endoscopic findings with leukocytosis (Fig. 5A) and an elevated serum GCSF concentration (Table 1).

We operated again and removed all of the evident liver and lymph node disease. Postoperatively, we used chemotherapy (with irinotecan [CPT-11] as the second-line agent and paclitaxel as the third-line drug) and the patient survived for almost 2 years before finally succumbing to liver failure after palliative radiation for bulky liver recurrence (Fig. 5B).

\section{Discussion}

Adenosquamous carcinoma of the stomach is very rare, accounting for fewer than $0.5 \%$ of all gastric cancers, and it tends to have more aggressive clinicopathologic features than adenosquamous carcinoma at other sites,

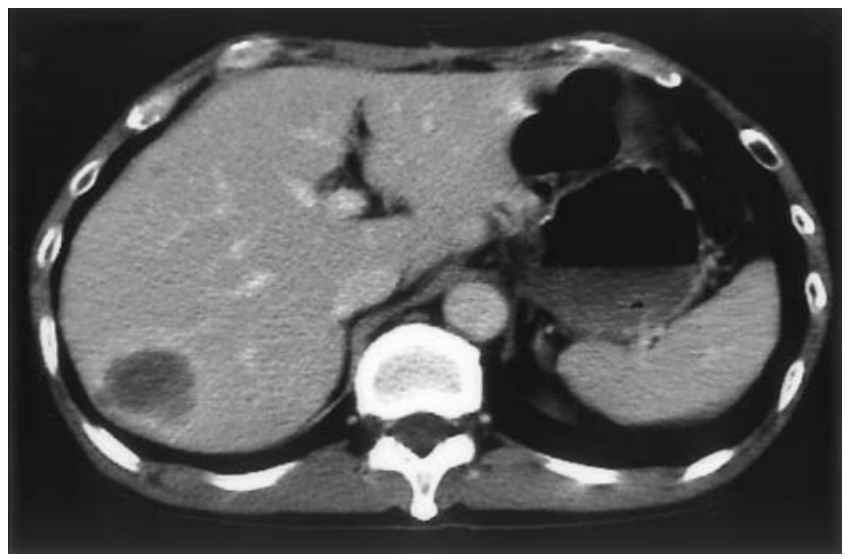

Fig. 4. Computed tomography indicated early recurrence in liver after curative gastrectomy

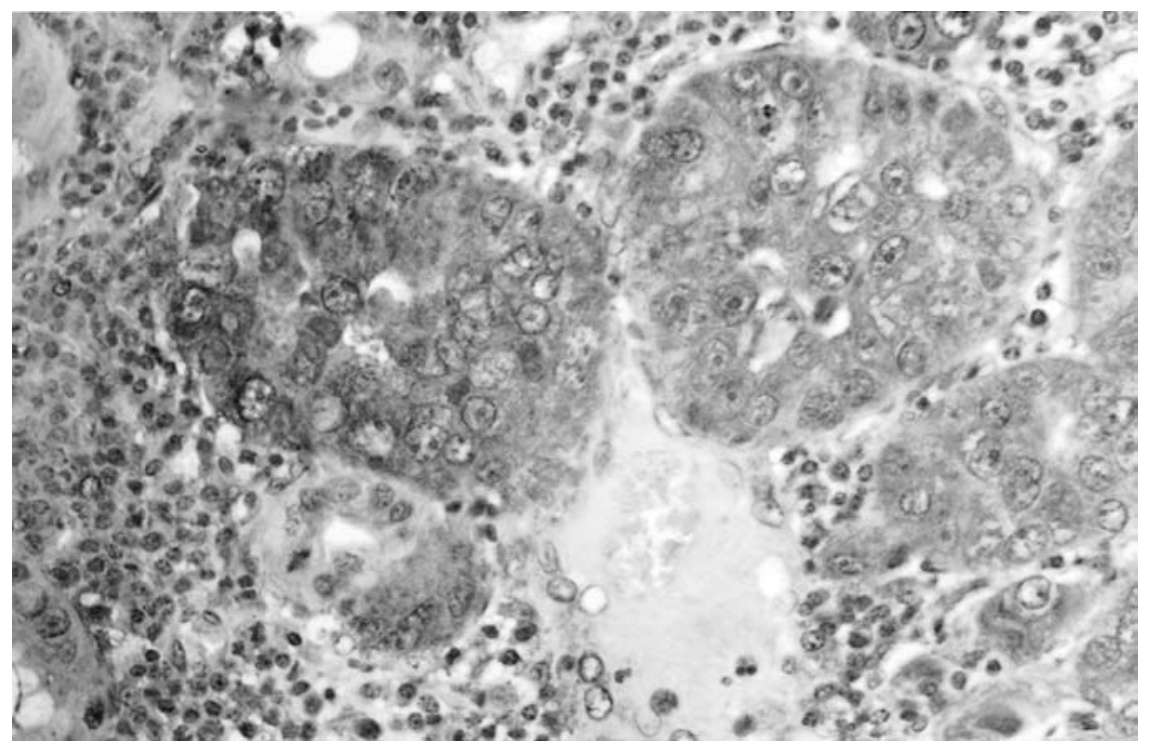

Fig. 3. Tumor cells stain positive with monoclonal antibody against granulocyte colony-stimulating factor (G-CSF; 4A6) 


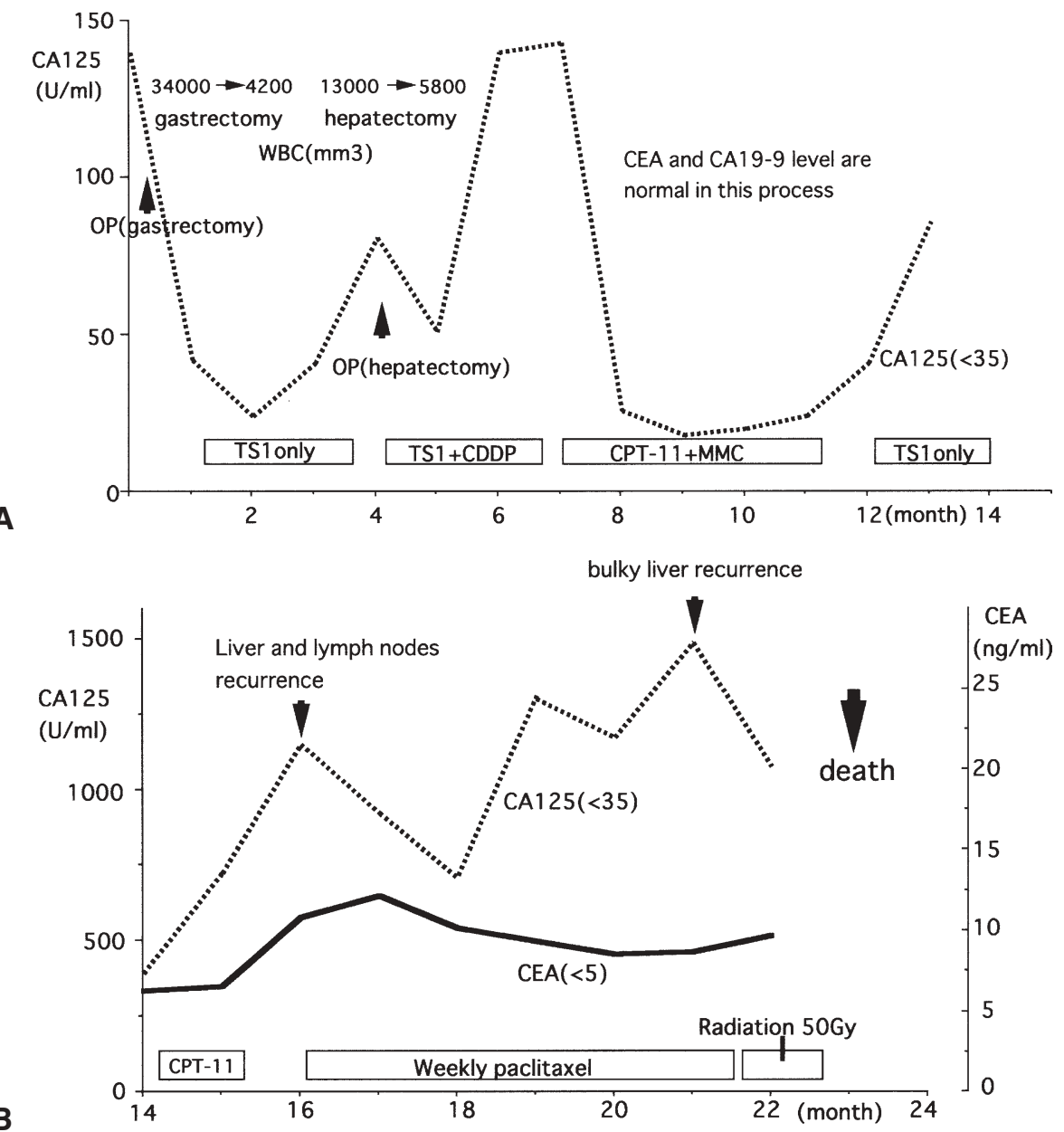

Fig. 5A,B. Postoperative chemotherapy and disease progression according to tumor markers; A until second-line chemotherapy; B from third-line chemotherapy. $C A 125$, carbohydrate antigen 125; $C E A$, carcinoembryonic antigen; $O P$, operation; TS1, S-1; CDDP, cisplatin; $C P T-11$, irinotecan; $M M C$, mitomycin $\mathrm{C}$ and a poor prognosis [9]. Macroscopically, most tumors are type 2 or 3 advanced gastric cancer according to Borrmann's classification [10]. The most common site of metastasis is the liver, and gastric adenosquamous carcinoma is associated with a worse prognosis than the more common types of adenocarcinoma. The poorer prognosis is probably related to the depth of tumor invasion and the relatively high frequency of lymphovascular invasion by carcinoma cells. Although G-CSF production has been found in cancers of the lung, gallbladder, liver, esophagus, and thyroid, there have been only a few reports about G-CSF-producing gastric cancers. G-CSF, a hematopoietic cytokine, regulates the proliferation and differentiation of granulocytic progenitor cells and functionally activates mature neutrophils. It also affects nonhematopoietic tumor cells by binding to G-CSF-specific receptors on their surfaces. G-CSF receptor expression by nonhematopoietic cancer cells may play a crucial role in mediating increasingly malignant behaviors [11]; autocrine growth promotion of malignant tumor cells by G-CSF has been suggested in human transitional carcinoma of the bladder [12].
In the present case, two rare factors coexisted in a patient - G-CSF production by a gastric adenosquamous carcinoma - which resulted in early recurrent disease of liver and lymph nodes just 3 months after curative gastrectomy with adjuvant chemotherapy. We performed a second operation for the recurrent disease and succeeded in resecting all visible lesions. Abnormally high levels of serum G-CSF and leukocytosis without evidence of infection were recognized before that procedure, which suggested the return of the G-CSF producing tumor; all abnormal signs disappeared postoperatively.

After the second operation, we prescribed extensive chemotherapy for the patient, with CPT-11 as the second-line and paclitaxel as the third-line agent, and this treatment produced a comparatively stable period for almost 1.5 years, except for the development of slight paraaortic lymphadenopathy and recurrent liver disease. Leukocytosis was absent during this period. Even in the last month, we did not identify marked leukocytosis, even though bulky tumors in the liver had appeared. These late findings may indicate that the GCSF-producing character of the tumor was lost after the 
second operation. Recent studies report that G-CSF protects malignant and nonmalignant cells from apoptosis induced by some factors, and the constitutive expression of G-CSF may protect tumors against an unfavorable environment $[13,14]$.

We suggest that the two coexisting malignant factors in our patient were removed by the first and second operations, which enabled us to achieve a comparatively stable period for him. Thus, even in a rare case of gastric adenosquamous carcinoma, close follow-up and use of adequate surgery and extensive chemotherapy may improve the patient's quality of life and prognosis for survival.

\section{References}

1. Kimura N, Niho Y, Yanase T. A high level of colony-stimulating activity in a lung cancer patient with extensive leucocytosis and the establishment of a CAS-producing cell line. Scand J Hematol 1982;28:417-24.

2. Egami H, Sakamoto K, Yoshimura R. Establishment of a cell line of gallbladder carcinoma producing human colony stimulating factor. Jpn J Cancer Res 1986;77:168-76.

3. Gabrilove J, Welte K, Lu L, Castro-Malaspina H, Moore MA. Constitutive production of leukemia differentiation colonystimulating erythroid burst-promoting and pluripoietic factors by a human hepatoma cell line. Blood 1985;66:407-15.

4. Ichiishi E, Yoshikawa T, Kogawa T, Yoshida N, Kondo M. Possible paracrine growth of adenocarcinoma of the stomach induced by granulocyte colony stimulating factor produced by squamous cell carcinoma of the esophagus. Gut 2000;46:432-4.
5. Kitamura H, Kodama F, Odagiri S, Nagahara N, Inoue $T$, Kanisawa M. Granulocytosis associated with malignant neoplasms. Hum Pathol 1989;20: 878-85.

6. Wu M, Cini JK, Yunis AA. Purification of a colony stimulating factor from cultured pancreatic carcinoma cells. J Biol Chem 1979;254:6626-8.

7. Okabe T, Nomura H, Osawa N. Establishment and characterization of a human colony-stimulating factor producing cell line from a squamous cell carcinoma of the thyroid gland. J Natl Cancer Inst 1982;69:1235-43.

8. Japanese Gastric Cancer Association. Japanese classification of gastric carcinoma, second English edition. Gastric Cancer 1998;1: $10-24$.

9. Mori M, Iwashita A, Enjoji M. Adenosquamous carcinoma of the stomach: a clinico-pathologic analysis of 28 cases. Cancer 1986;57: 333-9.

10. Boswell JT, Helwig EB. Squamous cell carcinoma and adenoacanthoma of the stomach. Cancer 1965;18:181-92.

11. Uzumaki H, Okabe T, Sasaki N, Hagiwara K, Takaku F, Tobita M, et al. Identification and characterization of receptors for granulocyte colony-stimulating factor on human placenta and trophoblastic cells. Proc Natl Acad Sci USA 1989;86:93236.

12. Tachibana M, Miyakawa A, Tazaki H, Nakamura K, Kubo A, Hata J, et al. Autocrine growth of transitional cell carcinoma of the bladder induced by granulocyte-colony stimulating factor. Cancer Res 1995;55:3438-43.

13. Wislez M, Fleury-Feith J, Rabbe N, Moreau J, Cesari D, Milleron $\mathrm{B}$, et al. Tumor-derived granulocyte macrophage stimulating factor and granulocyte colony stimulating factor prolong the survival of neutrophils infiltrating bronchoalveolar subtype pulmonary adenocarcinoma. Am J Pathol 2001;159:1423-33.

14. Uemura Y, Kobayashi M, Nakata H, Harada R, Kubota T, Taguchi $\mathrm{H}$. Effect ofserum deprivation on constitutive production of granulocyte-colony stimulatingfactor and granulocyte macrophage-colony stimulating factor in lung cancer cells. Int $\mathrm{J}$ Cancer 2004;109:826-32. 